Draft Version September 11, 2019

Preprint typeset using $\mathrm{L}^{A} \mathrm{~T}_{\mathrm{E}} \mathrm{X}$ style emulateapj v. 12/16/11

\title{
GAS AND DUST TEMPERATURE IN PRE-STELLAR CORES REVISITED: NEW LIMITS ON COSMIC-RAY IONIZATION RATE
}

\author{
Alexei V. Ivlev ${ }^{1}$, Kedron Silsbee ${ }^{1}$, Olli Sipilä ${ }^{1}$, Paola Caselli ${ }^{1}$ \\ ${ }^{1}$ Max-Planck-Institut für Extraterrestrische Physik, 85748 Garching, Germany \\ Draft version September 11, 2019
}

\begin{abstract}
We develop a self-consistent model for the equilibrium gas temperature and size-dependent dust temperature in cold, dense pre-stellar cores, assuming an arbitrary power-law size distribution of dust grains. Compact analytical expressions applicable to a broad range of physical parameters are derived and compared with predictions of the commonly used standard model. It is suggested that combining the theoretical results with observations should allow us to constrain the degree of dust evolution and the cosmic-ray ionization rate in dense cores, and to help in discriminating between different regimes of cosmic-ray transport in molecular clouds. In particular, assuming a canonical MRN distribution of grain sizes, our theory demonstrates that the gas temperature measurements in the pre-stellar core L1544 are consistent with an ionization rate as high as $\sim 10^{-16} \mathrm{~s}^{-1}$, an order of magnitude higher than previously thought.

Subject headings: ISM: clouds - dust, extinction - cosmic rays
\end{abstract}

\section{INTRODUCTION}

Pre-stellar cores set the initial conditions for the process of star formation (see, e.g., Shu et al. 1987; Bergin \& Tafalla 2007), and therefore determine the properties of the future stars and stellar systems which will form in their centers. Unveiling the physical and chemical structure of the cores puts stringent constraints on dynamical/chemical models (e.g., Keto et al. 2014 2015, Vasyunin et al. 2017, Sipilä \& Caselli 2018; Caselli et al. 2019). Specifically, measuring volume density and gas/dust temperature profiles is crucial to provide information about the heating by the interstellar radiation field (ISRF) (e.g., Zucconi et al. 2001, Launhardt et al. 2013, Steinacker et al. 2016 Harju et al. 2017; Hocuk et al. 2017) and cosmic rays (CRs) (e.g., Goldsmith 2001; Keto \& Caselli 2010), as well as about dust evolution (Sadavoy et al. 2016 Chacón-Tanarro et al. 2019). These processes critically affect the dynamical and chemical evolution of pre-stellar cores, as they regulate the ionisation fraction (e.g., McKee 1989) and surface chemistry/freeze-out rates (e.g., Zhao et al. 2018; Shingledecker et al. 2018).

The physical characteristics of pre-stellar cores exhibit significant variations. Typical values of the gas density $n_{\mathrm{g}}$ in the center of pre-stellar cores exceed $\sim 10^{5} \mathrm{~cm}^{-3}$ (e.g., Keto \& Caselli 2008; Keto et al. 2015). The gas cooling in this case is determined by collisions with dust grains, while the direct cooling by CO line emission (dominating at lower densities) becomes inefficient (Goldsmith 2001, Galli et al. 2002). Densities of up to $\sim 10^{7} \mathrm{~cm}^{-3}$ have been reported (Keto \& Caselli 2010 Caselli et al. 2019), while the actual peak density in contracting cores is expected to reach much higher values before a protostar forms. The magnitude of the CR ionization rate $\zeta_{\text {ion }}$ measured in the outer envelopes of molecular clouds (in a range of the gas column densi-

e-mail: ivlev@mpe.mpg.de

e-mail: ksilsbee@mpe.mpg.de ties around $N \sim 10^{21} \mathrm{~cm}^{-2}$ ) varies significantly from one object to another (Indriolo \& McCall 2012; Neufeld \& Wolfire 2017; Bacalla et al. 2019). Given uncertainties in the leading transport regime(s) governing the CR penetration into the clouds (Ivlev et al. 2018; Silsbee \& Ivlev 2019), this introduces significant uncertainty in the value of $\zeta_{\text {ion }}$ near the center; available theories (Padovani et al. 2018b) predict $\zeta_{\text {ion }} \sim 10^{-17}-10^{-16} \mathrm{~s}^{-1}$ for $N \sim 10^{23} \mathrm{~cm}^{-2}$.

The usual approach to calculate the gas and dust temperatures in dense cores (see, e.g., Zucconi et al. 2001 Hocuk et al. 2017, Chacón-Tanarro et al. 2019) relies on the assumption that the temperature of dust grains, $T_{\mathrm{d}}$, is determined from the balance of their radiative heating (by absorbing far-IR interstellar radiation penetrating into the cores) and cooling (via the continuum emission). Below we refer to this approach as the standard model, where the gas temperature $T_{\mathrm{g}}$ is controlled by the CR heating and cooling on the dust surface, proportional to the difference $T_{\mathrm{g}}-T_{\mathrm{d}}$. The available analytical models (e.g., Goldsmith 2001; Galli et al. 2002, Galli \& Padovani 2015) usually make further simplitication, assuming the dust grains to be monodisperse, i.e., to all have the same size. Even though the term describing the gas-dust thermal coupling is added to the dust energy balance in certain cases (Goldsmith 2001; Woitke et al. 2009, Akimkin et al. 2013), to the best of our knowledge such calculations never account for an explicit dependence of the resulting dust temperature on the grain size. At the same time, this dependence immediately follows from the very fact that the thermal coupling term is proportional to the grain area, while the radiative absorption and emission terms scale with the grain volume in the Rayleigh-Jeans regime.

The aim of the present paper is to develop a selfconsistent analytical model for the equilibrium gas temperature and size-dependent dust temperature in dense pre-stellar cores, depending on a given local gas density, local radiation field and the $\mathrm{CR}$ ionization rate, and a 
power-law size distribution of dust grains. We derive compact expressions applicable for typical conditions in dense cores, and compare our results with predictions of the commonly used standard model. Combined with observations, our findings should have important implications - in particular, for constraining the CR ionization rate and the degree of dust evolution in dense cores, for discriminating between different regimes of CR transport in molecular clouds, and for estimating the speed of physical and chemical processes occurring on the surface of grains.

\section{HEATING AND COOLING IN DENSE CORES}

In this section we summarize the main heating and cooling mechanisms of gas and dust, relevant to dense pre-stellar cores, and formulate the respective balance equations.

\subsection{Balance equation for dust}

The UV and visible radiation of the ISRF are practically completely attenuated in dense cores (see, e.g., Hocuk et al. 2017, and references therein). Therefore, dust is heated by absorbing photons from the far-IR part of the spectrum, still able to penetrate into the cores. ${ }^{1}$ This is usually assumed to be the only heating mechanism, which is balanced by the modified black-body radiative cooling of dust grains. In the present paper, we also include the effect of the gas-dust thermal coupling. This leads to additional collisional heating due to gas particles impinging on the dust surface. ${ }^{2}$ Then the energy balance for a dust grain can be written in the following form (Goldsmith 2001):

$$
\dot{E}_{\mathrm{em}}-\dot{E}_{\mathrm{abs}}=\dot{E}_{\mathrm{gd}},
$$

where $\dot{E}_{\text {em }}$ and $\dot{E}_{\text {abs }}$ are the rates of the radiative cooling (emission) and heating (absorption), and $\dot{E}_{\mathrm{gd}}$ is the rate of collisional heating.

The rate of radiative heating of a grain of radius $a$ is (Draine 2011)

$$
\dot{E}_{\mathrm{abs}}=\pi a^{2} c \int u_{\nu}(N) Q_{\mathrm{abs}}(\nu, a) d \nu
$$

where $u_{\nu}(N)$ is the specific energy density of the ISRF at the gas column density $N, Q_{\text {abs }}(\nu, a)$ is the dust absorption efficiency for the frequency $\nu$, and $c$ is the speed of light. For the radiation dominated by far-IR photons $Q_{\text {abs }}(\nu, a) \propto \nu^{2} a$ (the spectral index of the dust opacity is assumed to be equal to 2). Hence, $E_{\text {abs }}=f(N) a^{3}$, where $f(N)$ is a function of $N$.

The rate of radiative cooling is (Draine 2011)

$$
\dot{E}_{\mathrm{em}}=4 \pi a^{2}\left\langle Q_{\mathrm{abs}}(\nu, a)\right\rangle_{T_{\mathrm{d}}} \sigma T_{\mathrm{d}}^{4}(a) .
$$

Here, $\left\langle Q_{\mathrm{abs}}\right\rangle_{T_{\mathrm{d}}}=q_{\mathrm{abs}} T_{\mathrm{d}}^{2} a$ is the Planck-averaged absorption efficiency of dust, where $T_{\mathrm{d}}$ is the size-dependent dust temperature, $\sigma$ is the Stefan-Boltzmann constant, and $q_{\text {abs }}$ is a material-dependent numerical factor, equal

\footnotetext{
1 As discussed by Zucconi et al. (2001), UV and optical radiation is converted into far- $I \mathrm{~K}$ radiation in a photodissociation layer surrounding the core. This could contribute to the far-IR heating, but typically is not taken into account in radiative transfer models.

2 The thermal coupling always leads to dust heating if it is the only mechanism of gas cooling (see Section 2.2).
}

to $\approx 0.13 \mathrm{~K}^{-2} \mathrm{~cm}^{-1}$ for silicate grains (we assume this value for calculations below). We obtain

$$
\dot{E}_{\mathrm{em}}=4 \pi q_{\mathrm{abs}} \sigma T_{\mathrm{d}}^{6}(a) a^{3} .
$$

Then the radiative heating rate can be conveniently presented in the following identical form:

$$
\dot{E}_{\mathrm{abs}} \equiv 4 \pi q_{\mathrm{abs}} \sigma T_{\mathrm{d} 0}^{6}(N) a^{3},
$$

where $T_{\mathrm{d} 0}$ is the local equilibrium dust temperature from the absorption-emission balance, i.e., when $\dot{E}_{\mathrm{gd}}$ in Equation (1) is neglected.

The gas-dust thermal coupling is characterized by the rate of collisional heating of a dust grain (Draine 2011 Burke \& Hollenbach 1983),

$$
\dot{E}_{\mathrm{gd}}=4 \sqrt{2 \pi} a^{2} \alpha_{\mathrm{g}} n_{\mathrm{g}} v_{\mathrm{g}}^{*} k_{\mathrm{B}}\left[T_{\mathrm{g}}-T_{\mathrm{d}}(a)\right],
$$

where $T_{\mathrm{g}}$ is the gas temperature, $v_{\mathrm{g}}^{*}$ is the thermal velocity scale of gas particles, $\alpha_{\mathrm{g}}$ is their thermal accommodation coefficient on dust surface, and $k_{\mathrm{B}}$ is Boltzmann's constant. To include the effect of the ISM elemental composition, we set the density of $\mathrm{H}_{2}$ molecules as the relevant gas density scale $n_{\mathrm{g}}$. Then the velocity scale,

$$
v_{\mathrm{g}}^{*}=\left(1+2 \sqrt{2} \sum_{i} \frac{x_{i}}{\sqrt{\mu_{i}}}\right) v_{\mathrm{g}} \approx 1.14 v_{\mathrm{g}},
$$

is determined by the mass numbers $\mu_{i}$ of heavier elements and their abundances $x_{i}$ (with respect to the atomic hydrogen), with $v_{\mathrm{g}}=\sqrt{k_{\mathrm{B}} T_{\mathrm{g}} / m_{\mathrm{g}}}$ being the thermal velocity scale of $\mathrm{H}_{2}$ molecules. For the accommodation coefficient, we adopt $\alpha_{\mathrm{g}} \approx 0.5$ (Draine 2011).

\subsection{Balance equation for gas}

The gas heating in pre-stellar cores is completely dominated by CRs, whereas the cooling occurs through two mechanisms (Goldsmith 2001; Galli et al. 2002): Apart from the gas-dust thermal coupling, the molecular lines may contribute to the cooling. The main line coolant in dense cores is the low- $J$ rotational transitions of CO molecules, whose catastrophic freeze-out makes this mechanism unimportant for typical densities in the center of dense cores (Caselli et al. 1999, Goldsmith 2001). Using the gas-grain chemical model by Sipilä et al. (2019) in conjunction with the hydrodynamical model by Sipilä \& Caselli (2018), we have concluded that the line cooling can be safely neglected for $n_{\mathrm{g}} \gtrsim 10^{5} \mathrm{~cm}^{-3}$, and then we can write the thermal balance as

$$
\Gamma_{\mathrm{CR}}=\Lambda_{\mathrm{gd}} .
$$

Here, $\Gamma_{\mathrm{CR}}$ is the $\mathrm{CR}$ heating rate and $\Lambda_{\mathrm{gd}}$ is the cooling function due to gas-dust thermal coupling.

The heating rate per unit volume can be presented in the following form (Glassgold et al. 2012):

$$
\Gamma_{\mathrm{CR}}=\zeta_{\text {ion }}(N) \varepsilon_{\text {heat }}\left(n_{\mathrm{g}}\right) n_{\mathrm{g}},
$$

where $\zeta_{\text {ion }}$ is the total $\mathrm{CR}$ ionization rate per $\mathrm{H}_{2}$ molecule and $\varepsilon_{\text {heat }}$ is the heating energy per $\mathrm{H}_{2}$ ionization. The latter is a very slowly increasing function of $n_{\mathrm{g}}$, reaching values of $15-17 \mathrm{eV}$ for dense cores; below we adopt the value of $\varepsilon_{\text {heat }}=16 \mathrm{eV}$. 
The gas cooling function is determined by the rate of dust collisional heating,

$$
\Lambda_{\mathrm{gd}}=\int \dot{E}_{\mathrm{gd}} d n_{\mathrm{d}}
$$

We generally assume an "evolved MRN" size distribution of grains,

$$
\frac{d n_{\mathrm{d}}}{d a} \propto a^{-3.5+\gamma},
$$

in the range of $a_{\min } \leq a \leq a_{\max }$. The deviation of $a_{\min }$ and $a_{\max }$ from the "canonical" MRN values as well as nonzero $\gamma$ parameterize the degree of dust evolution due to possible coagulation in cores (Weingartner \& Draine 2001). The value of $a_{\max }$ is not expected to exceed a few tenths of $\mu \mathrm{m}$, while $a_{\text {min }}$ may increase significantly due to efficient depletion of smaller grains onto bigger ones. The slope variation is usually positive and could be as large as $\gamma \approx 1$ (or even larger). The scale factor in Equation (8) is determined from the relation between the mass densities of dust and gas,

$$
f_{\mathrm{d}}^{*} m_{\mathrm{g}} n_{\mathrm{g}}=\frac{4}{3} \pi \rho_{\mathrm{d}} \int_{a_{\min }}^{a_{\max }} a^{3} \frac{d n_{\mathrm{d}}}{d a} d a .
$$

Here, $\rho_{\mathrm{d}}$ is the mass density of grain material, equal to $\approx 3.5 \mathrm{~g} \mathrm{~cm}^{-3}$ for compact silicate, and $f_{\mathrm{d}}^{*}$ is the dust-togas mass ratio where the normalization is by $m_{\mathrm{g}} n_{\mathrm{g}}$,

$$
f_{\mathrm{d}}^{*}=\left(1+\sum_{i} x_{i} \mu_{i}\right) f_{\mathrm{d}} \approx 1.4 f_{\mathrm{d}},
$$

expressed in terms of the standard value of $f_{\mathrm{d}}=0.01$. Below we adopt these values of $\rho_{\mathrm{d}}$ and $f_{\mathrm{d}}$.

We stress that the absorption term in Equation (1) is completely determined by the local density of the ISRF, i.e., the coupling to the local thermal radiation of dust is neglected. This approach is justified provided the optical depth of the core is small for the thermal radiation. Using the above expression for $\left\langle Q_{\mathrm{abs}}\right\rangle_{T_{\mathrm{d}}}$, we obtain $\approx \pi q_{\text {abs }} T_{\mathrm{d}}^{2} a^{3}$ for the corresponding absorption cross section. Then, utilizing relation (9) and the fact that the cross section scales with $a^{3}$, we readily infer the optical depth, $\tau_{\text {core }} \approx \frac{3}{4}\left(q_{\text {abs }} f_{\mathrm{d}}^{*} T_{\mathrm{d}}^{2} / \rho_{\mathrm{d}}\right) \Sigma_{\text {core }}$, where $\Sigma_{\text {core }}=m_{\mathrm{g}} N_{\text {core }}$ is the $\mathrm{H}_{2}$ surface density of the core. For cold dense cores with $T_{\mathrm{d}} \approx 6 \mathrm{~K}$ and the column density of $N_{\text {core }} \sim 10^{23} \mathrm{~cm}^{-2}$ Crapsi et al. 2007, Keto \& Caselli 2010 ) we estimate $\tau_{\text {core }} \sim 3 \times 10^{-3}$. As the peak column density is expected to approximately scale with the square root of the peak volume density (for the BonnorEbert sphere, Bonnor 1956), our approach should be well applicable for $n_{\mathrm{g}}$ of up to $\sim 10^{10} \mathrm{~cm}^{-3}$.

\subsection{Additional mechanisms of $C R$ heating}

To facilitate the analysis and comparison with previous results, above we only discussed the heating included in the standard model for the equilibrium gas and dust temperatures. Apart from this, there are additional mechanisms of dust and gas heating by CRs, associated with the presence of dust grains. The dust heating is due to direct CR bombardment, leading to the energy deposition into the grains (e.g., Léger et al. 1985, Shen et al. 2004), and due to absorption of UV radiation caused by the CR-induced fluorescence of $\mathrm{H}_{2}$ and $\mathrm{He}$ (Prasad \&
Tarafdar 1983: Cecchi-Pestellini \& Aiello 1992). Near-IR radiation from vibrationally excited $\mathrm{H}_{2}$ may add to dust heating (Dalgarno et al. 1999), while the UV radiation also contributes to gas heating via photoelectric emission from dust (Draine 1978). Furthermore, CRs are the only source of atomic hydrogen in dense cores (Padovani et al. 2018a). The energy released in the recombination on the surface of grains is distributed between dust and gas.

The mechanisms of dust heating by CRs require a careful analysis, which is presented in Appendix A. We show that for typical conditions in dense pre-stellar cores (discussed in Section 4.4 this heating is unimportant.

The effect of additional gas heating by CRs is straightforwardly included in Equations (5) and (6), by adding the corresponding energy to $\varepsilon_{\text {heat }}$. The upper bound for the energy of photoelectric heating can be estimated as a product of the photoelectric yield and the energy of CRinduced UV heating of dust. With the energy of $\approx 8 \mathrm{eV}$ (Dalgarno et al. 1999) and the yield of $\sim 0.1$ for LymanWerner photons and MRN silicate grains (Weingartner et al. 2006), we obtain the photoelectric heating energy of less than $1 \mathrm{eV}$ per $\mathrm{H}_{2}$ ionization. The gas heating due to hydrogen recombination, although uncertain, is expected to give a comparable contribution (Glassgold et al. 2012). As these energies are substantially smaller than the adopted value of $\varepsilon_{\text {heat }}$, we conclude that the additional gas heating can also be neglected.

\section{EQUILIBRIUM TEMPERATURES}

Substituting Equations (2)-4 in Equation (1), we get the thermal balance equation for dust grains:

$$
\left[T_{\mathrm{d}}^{6}(a)-T_{\mathrm{d} 0}^{6}\right] a=\sqrt{\frac{2}{\pi}} \frac{\alpha_{\mathrm{g}} n_{\mathrm{g}} v_{\mathrm{g}}^{*}}{q_{\mathrm{abs}} \sigma} k_{\mathrm{B}}\left[T_{\mathrm{g}}-T_{\mathrm{d}}(a)\right] .
$$

The complementary balance equation for gas particles is obtained from Equation (5): To derive $\Lambda_{\mathrm{gd}}$, we insert Equation (4) in Equation (7), and calculate the scale factor for the size distribution (8). Substituting the result in Equation (5) and using Equation (6), we get

$$
\begin{gathered}
\zeta_{\text {ion }}=\sqrt{\frac{18}{\pi}} \frac{\alpha_{\mathrm{g}} f_{\mathrm{d}}^{*} m_{\mathrm{g}} n_{\mathrm{g}} v_{\mathrm{g}}^{*}}{\varepsilon_{\text {heat }} \rho_{\mathrm{d}}} \frac{0.5+\gamma}{a_{\max }^{0.5+\gamma}-a_{\min }^{0.5+\gamma}} \\
\quad \times \int_{a_{\min }}^{a_{\max }} k_{\mathrm{B}}\left[T_{\mathrm{g}}-T_{\mathrm{d}}(a)\right] a^{-1.5+\gamma} d a .
\end{gathered}
$$

Equations 10 and 11 yield the self-consistent solution for $T_{\mathrm{d}}(a)$ and $T_{\mathrm{g}}$ for given local ionization rate and local conditions in the core.

Below we give the analytical approximation of Equations (10) and (11), allowing us to derive the explicit dependence of the dust temperature on the grain size and better understand the mechanism behind the observed behavior.

\subsection{Analytical approximation}

Assume $\Delta T_{\mathrm{d}}(a) \equiv T_{\mathrm{d}}(a)-T_{\mathrm{d} 0}$ is sufficiently small, so that the lhs of Equation 10 can be expanded in a series over $\Delta T_{\mathrm{d}}$. Keeping up to quadratic terms, $\approx 6 T_{\mathrm{d} 0}^{5} \Delta T_{\mathrm{d}}+$ $15 T_{\mathrm{d} 0}^{4} \Delta T_{\mathrm{d}}^{2}$, ensures accurate results for $\Delta T_{\mathrm{d}} / T_{\mathrm{d} 0} \lesssim 2 / 5$ and leads to a quadratic equation for $\Delta T_{\mathrm{d}}$. Keeping the same accuracy for $\Delta T_{\mathrm{g}}$, we obtain

$$
\Delta T_{\mathrm{d}}(a) \approx\left(1-\frac{5}{2} \frac{a / A}{(1+a / A)^{2}} \frac{\Delta T_{\mathrm{g}}}{T_{\mathrm{d} 0}}\right) \frac{\Delta T_{\mathrm{g}}}{1+a / A},
$$


where $\Delta T_{\mathrm{g}} \equiv T_{\mathrm{g}}-T_{\mathrm{d} 0}$ and

$$
A=\frac{1}{\sqrt{18 \pi}} \frac{\alpha_{\mathrm{g}} n_{\mathrm{g}} v_{\mathrm{g}}^{*} k_{\mathrm{B}}}{q_{\mathrm{abs}} \sigma T_{\mathrm{d} 0}^{5}},
$$

is the "critical" grain radius. For "overcritical" grains with $a \gg A$ we have $\Delta T_{\mathrm{d}}(a) \ll \Delta T_{\mathrm{g}}$, i.e., their temperature is practically equal to $T_{\mathrm{d} 0}$, as usually assumed; on the other hand, for grains with $a \lesssim A$ the thermal coupling to gas dominates their energy balance and, hence, their temperature approaches $T_{\mathrm{g}}$. As a consequence, the contribution of small grains to the gas cooling [determined by the integral in Equation (11)] can be reduced drastically, which should lead to higher gas temperatures compared to the case where $T_{\mathrm{d}}=T_{\mathrm{d} 0}$ is assumed.

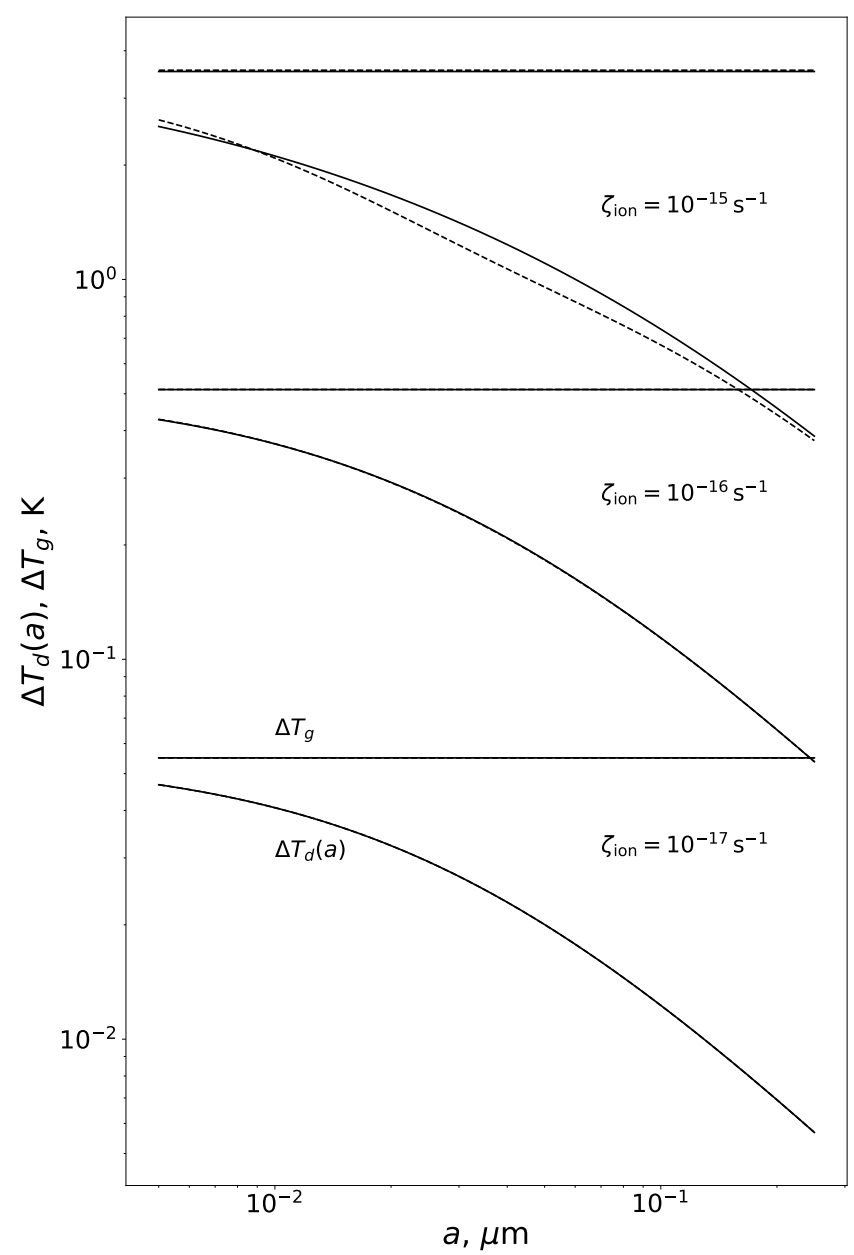

FIG. 1.- The excess of gas and dust temperature, $\Delta T_{\mathrm{g}}=$ $T_{\mathrm{g}}-T_{\mathrm{d} 0}$ and $\Delta T_{\mathrm{d}}(a)=T_{\mathrm{d}}(a)-T_{\mathrm{d} 0}$, above the temperature of the absorption-emission balance, $T_{\mathrm{d}}$. The three sets of solid lines illustrate the solution of Equations 10 and 11 for the three indicated values of the $\mathrm{CR}$ ionization rate $\zeta_{\text {ion }}$, plotted versus the dust grain radius $a$ (the horizontal lines show $\Delta T_{\mathrm{g}}$ ). The dashed lines (almost overlapped with the solid lines for $\Delta T_{\mathrm{g}}$ ) represent the analytical approximation: Equations $\sqrt{14}$ and $\sqrt{15}$ for $\Delta T_{\mathrm{g}}$ and Equation $(12)$ for $\Delta T_{\mathrm{d}}(a)$. The results are for $T_{\mathrm{d} 0}=6 \mathrm{~K}$, assuming the canonical MRN size distribution $\left(\gamma=0, a_{\min }=0.005 \mu \mathrm{m}\right.$, $\left.a_{\max }=0.25 \mu \mathrm{m}\right)$ and the gas density $n_{\mathrm{g}}=10^{6} \mathrm{~cm}^{-3}$.

The critical radius is determined by the materialdependent absorption factor $q_{\mathrm{abs}}$ and by the local con- ditions in the core (in particular, it depends on $T_{\mathrm{g}}$ via $v_{\mathrm{g}}^{*}$ ). Note that $A$ increases with $n_{\mathrm{g}}$ faster than linearly, since $T_{\mathrm{d} 0}$ is a decreasing function of $N$ (Evans et al. 2001 Hocuk et al. 2017, Chacón-Tanarro et al. 2019).

We introduce dimensionless parameters

$$
\tilde{R}=a_{\max } / a_{\text {min }}, \quad \tilde{A}=A_{0} / a_{\text {min }},
$$

where $A_{0} \equiv A\left(T_{\mathrm{g}}=T_{\mathrm{d} 0}\right)$. Parameter $\tilde{R}$ quantifies the relative width of the size distribution, while $\tilde{A}$ characterizes the relative importance of the critical radius: for $\tilde{A} \gtrsim 1$ the overall effect of finite $\Delta T_{\mathrm{d}}$ is expected to become significant. Substituting Equation (12) in Equation (11) and expanding $v_{\mathrm{g}}^{*}\left(T_{\mathrm{g}}\right)$ in a series over $\Delta T_{\mathrm{d}}$, after some manipulation we obtain:

$$
\zeta_{\text {ion }} \approx \sqrt{\frac{18}{\pi}} \frac{\alpha_{\mathrm{g}} f_{\mathrm{d}}^{*} m_{\mathrm{g}} n_{\mathrm{g}} v_{0}^{*} k_{\mathrm{B}} T_{\mathrm{d} 0}}{\varepsilon_{\text {heat }} \rho_{\mathrm{d}} a_{\text {min }}} \Psi\left(\Delta T_{\mathrm{g}}\right),
$$

where $v_{0}^{*} \equiv v_{\mathrm{g}}^{*}\left(T_{\mathrm{g}}=T_{\mathrm{d} 0}\right)$. Function $\Psi\left(\Delta T_{\mathrm{g}}\right)$ gives the explicit dependence on the temperature difference as well as on the dimensionless parameters of the size distribution,

$$
\Psi=\frac{0.5+\gamma}{\tilde{R}^{0.5+\gamma}-1}\left(I_{1}+I_{2} \frac{\Delta T_{\mathrm{g}}}{T_{\mathrm{d} 0}}\right) \frac{\Delta T_{\mathrm{g}}}{T_{\mathrm{d} 0}},
$$

where auxiliary functions $I_{1,2}(\tilde{A}, \tilde{R}, \gamma)$ are determined by Equation (B1) in Appendix B. Thus, Equation (14) provides us with a convenient direct relation between the gas temperature and the ionization rate.

In Figure 1 we compare the exact and analytical solutions. The solid lines represent the solution of Equations $(10)$ and (11) for three different values of $\zeta_{\text {ion }}$, showing $\Delta T_{\mathrm{d}}$ (as a function of grain radius) and the corresponding $\Delta T_{\mathrm{g}}$. We set $T_{\mathrm{d} 0}=6 \mathrm{~K}$ for the illustration, which is about the value expected from the absorptionemission balance in the center of very dense cores (assuming a typical ISRF, see, e.g., Crapsi et al. 2007). The results are obtained for the canonical MRN distribution and a gas density typical to such cores $\left(n_{\mathrm{g}}=10^{6} \mathrm{~cm}^{-3}\right.$, see, e.g., Crapsi et al. 2005), demonstrating that the temperature of smaller grains approaches $T_{\mathrm{g}}$, while for bigger grains it approaches $T_{\mathrm{d} 0}$. This trend is observed for all used values of $\zeta_{\text {ion }}$. According to Equation (12), the transition occurs at $a \approx A$, which is about $0.03 \mu \mathrm{m}$ for the chosen conditions. As the critical radius $A$ scales (faster than) linearly with the gas density, an increase in $n_{\mathrm{g}}$ by a factor of $\sim 10$ will make $T_{\mathrm{d}}(a)$ almost equal to $T_{\mathrm{g}}$ for grains below $a \sim 0.1 \mu \mathrm{m}$.

The analytical approximation is depicted in Figure 1 by the dashed lines. We see that Equations (14) and (15) provide excellent accuracy for the gas temperature for all values of $\zeta_{\text {ion }}$ used in calculations, so that the dashed and solid lines for $\Delta T_{\mathrm{g}}$ are undistinguishable. Some deviation of Equation (12) from the exact solution for $\Delta T_{\mathrm{d}}(a)$ (occurring at $a \sim A$ ) is only seen in the extreme case of $\zeta_{\text {ion }}=10^{-15} \mathrm{~s}^{-1}$.

\section{DISCUSSION}

Let us quantify the difference between the standard model for gas temperature and our model for $T_{\mathrm{g}}$ and $T_{\mathrm{d}}(a)$. To facilitate the analysis, we introduce a concept of effective grain radius and replace the self-consistent 
dependence $\Delta T_{\mathrm{d}}(a)$, given by Equation 10 , with an effective (size-independent) value $\Delta T_{\mathrm{d} \text {,eff }}$. The latter is derived by integrating Equation (1) over the size distribution (8) and assuming that $T_{\mathrm{d}}$ does not depend on $a$. The results of this approach should tend to exact results both for small $\tilde{A}$ (where the deviation of $T_{\mathrm{d}}$ from $T_{\mathrm{d} 0}$ is insignificant) and for sufficiently large $\tilde{A}$ (where all grains in the MRN size range have the temperature close to $T_{\mathrm{g}}$ ).

\subsection{Effective grain radius}

Following the steps of Sec. 3.1, we obtain the relation between $\Delta T_{\mathrm{d} \text {,eff }}$ and $\Delta T_{\mathrm{g}}$ in the form of Equation $[12$, where $a$ should be replaced with the effective grain radius $a_{\text {eff }}=a_{\text {min }} \tilde{a}_{\text {eff }}$. It is determined by

$$
\tilde{a}_{\mathrm{eff}}(\tilde{R}, \gamma)=\frac{-0.5+\gamma}{\tilde{R}^{-0.5+\gamma}-1} \frac{\tilde{R}^{0.5+\gamma}-1}{0.5+\gamma},
$$

a monotonically increasing function of both variables. For $\gamma \geq 0$, it is bound between $\tilde{R}^{0.5} \leq \tilde{a}_{\text {eff }}<\tilde{R}$, i.e., $\left(a_{\min } a_{\max }\right)^{0.5} \leq a_{\mathrm{eff}}<a_{\max }$. The lower bound corresponds to $\gamma=0$; for $\gamma=0.5$ and $\gamma=1$ we have $\tilde{a}_{\text {eff }}=(\tilde{R}-1) / \ln \tilde{R}$ and $\tilde{a}_{\text {eff }}=\left(\tilde{R}+\tilde{R}^{0.5}+1\right) / 3$, respectively. The case of monodisperse grains is naturally recovered for $\tilde{R} \rightarrow 1$, where $a_{\text {eff }} \rightarrow a_{\mathrm{min}}$. As discussed in Section 4.2, the value of $a_{\text {eff }}$ plays the critical role in determining the value of $\Delta T_{\mathrm{g}}$.

The gas temperature is then derived from Equation (14) where $\Psi$ should be replaced with the corresponding effective function,

$$
\begin{aligned}
& \Psi_{\mathrm{eff}}=\frac{1 / \tilde{a}_{\mathrm{eff}}}{1+\tilde{A} / \tilde{a}_{\mathrm{eff}}} \\
& \times\left[1+\frac{1}{2}\left(\frac{1}{1+\tilde{A} / \tilde{a}_{\mathrm{eff}}}+\frac{5}{\left(1+\tilde{a}_{\mathrm{eff}} / \tilde{A}\right)^{2}}\right) \frac{\Delta T_{\mathrm{g}}}{T_{\mathrm{d} 0}}\right] \frac{\Delta T_{\mathrm{g}}}{T_{\mathrm{d} 0}} .
\end{aligned}
$$

Let us analyze Equation (17). In the limit of small $A_{0} / a_{\text {eff }}$ (where the deviation of the dust temperature from $T_{\mathrm{d} 0}$ is negligible), the factor in the brackets is $\approx \sqrt{T_{\mathrm{g}} / T_{\mathrm{d} 0}}$ for the accepted accuracy. This yields

$$
A_{0} \ll a_{\text {eff }}: \quad \zeta_{\text {ion }} \approx \sqrt{\frac{18}{\pi}} \frac{\alpha_{\mathrm{g}} f_{\mathrm{d}}^{*} m_{\mathrm{g}} n_{\mathrm{g}}}{\varepsilon_{\text {heat }} \rho_{\mathrm{d}} a_{\mathrm{eff}}} v_{\mathrm{g}}^{*}\left(T_{\mathrm{g}}\right) k_{\mathrm{B}} \Delta T_{\mathrm{g}} .
$$

We see that Equation (18) represents the standard model for the gas temperature (Goldsmith 2001, Galli et al. 2002 ), which is now generalized to the case of arbitrary power-law size distribution with the effective grain radius $a_{\text {eff }}$.

The absorption-emission balance assumed in the standard model is completely invalid for large $A_{0} / a_{\text {eff }}$ (where the effective dust temperature substantially exceeds $\left.T_{\mathrm{d} 0}\right)$. The second line of Equation (17) in this limit represents the first two terms of expansion of $\left(T_{\mathrm{g}} / T_{\mathrm{d} 0}\right)^{6}-1$ over $\Delta T_{\mathrm{g}}$. With the same accuracy, we can write ${ }^{3}$

$$
A_{0} \gg a_{\text {eff }}: \quad \zeta_{\text {ion }} \approx 3 \frac{f_{\mathrm{d}}^{*} m_{\mathrm{g}} q_{\mathrm{abs}} \sigma}{\varepsilon_{\text {heat }} \rho_{\mathrm{d}}}\left(T_{\mathrm{g}}^{6}-T_{\mathrm{d} 0}^{6}\right) .
$$

3 This expression can be readily obtained from Equation 1 by integrating the latter over the size distribution and equating the resulting rhs to $\Gamma_{\mathrm{CR}}$. Then, assuming $T_{\mathrm{d}} \approx T_{\mathrm{g}}$ and utilizing relation $\sqrt{90}$, we arrive to Equation 19.
For sufficiently small $\Delta T_{\mathrm{g}}$, the rhs of Equation 190 is a factor of $1+A_{0} / a_{\text {eff }}$ smaller than that of Equation (18), as it follows from Equation (17). This implies that $\Delta T_{\mathrm{g}}$ for $A_{0} \gg a_{\text {eff }}$ is by this factor larger than the prediction of the standard theory. Furthermore, the nonlinearity in Equation $(19)$ is substantially stronger than that in Equation (18), which is another consequence of the deviation of dust temperature from $T_{\mathrm{d} 0}$ (neglected in the standard model). A remarkable fact is that Equation (19) is explicitly independent of the gas density and characteristics of the size distribution [an implicit dependence on $n_{\mathrm{g}}$ is via $\left.\zeta_{\text {ion }}(N)\right]$. This is a natural consequence of strong gas-dust thermal coupling in the limit $A_{0} \gg a_{\text {eff }}$, so $\Delta T_{\mathrm{g}}$ is a nearly universal function of the column density.

Since the critical grain radius, Equation (13), is an increasing function of $n_{\mathrm{g}}$ (and $N$ ), a transition to large $A_{0} / a_{\text {eff }}$ occurs at a certain critical density. For the MRN size distribution, Equation $\sqrt{19}$ is applicable for $n_{\mathrm{g}}$ satisfying the condition $A_{0}\left(n_{\mathrm{g}}\right) \gg\left(a_{\min } a_{\max }\right)^{0.5}$, which requires gas densities substantially larger than $10^{6} \mathrm{~cm}^{-3}$.

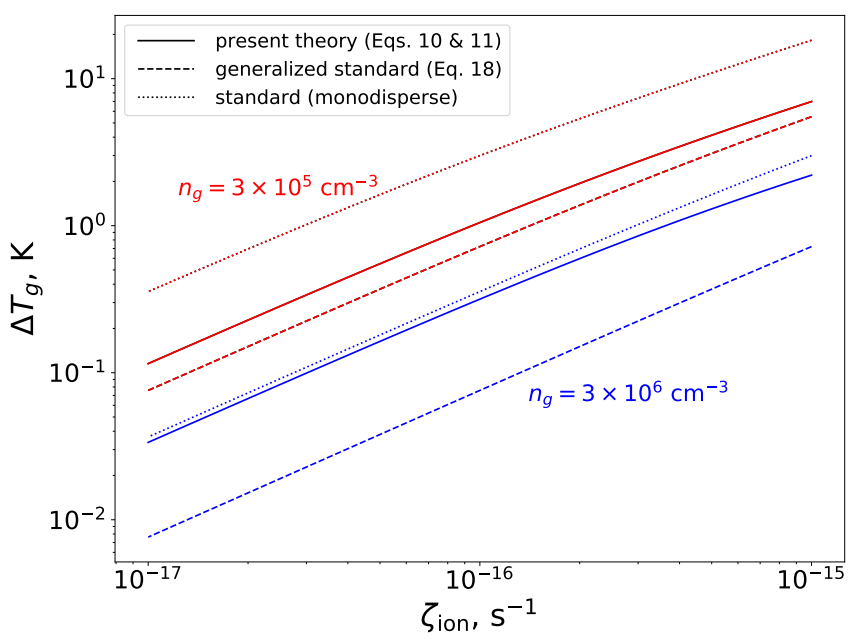

FIG. 2.- Gas temperature difference $\Delta T_{\mathrm{g}}$ as a function of the $\mathrm{CR}$ ionization rate $\zeta_{\text {ion }}$. The solid and dashed lines represent, respectively, the present theory (exact solution) and the prediction of the generalized standard theory, both derived for the MRN size distribution. The dotted line shows $\Delta T_{\mathrm{g}}$ calculated from the standard theory assuming monodisperse grains of $a=0.17 \mu \mathrm{m}$. The upper and lower sets of curves are for $n_{\mathrm{g}}=3 \times 10^{5} \mathrm{~cm}^{-3}$ and $3 \times 10^{6} \mathrm{~cm}^{-3}$, respectively, the other conditions as in Figure 1

Figure 2 summarizes results for the gas temperature, presenting $\Delta T_{\mathrm{g}}\left(\zeta_{\text {ion }}\right)$ for $n_{\mathrm{g}}=3 \times 10^{5} \mathrm{~cm}^{-3}$ and $3 \times 10^{6} \mathrm{~cm}^{-3}$. With the solid lines we plot the exact solution of Equations (10) and (11) [the analytical solution, Equations (14) and (15), is nearly indistinguishable], while the dashed lines depict the generalized standard model described by Equation (18). We see that the latter systematically underestimates $\Delta T_{\mathrm{g}}$. The disparity increases with gas density, as $\approx 1+A_{0}\left(n_{\mathrm{g}}\right) / a_{\mathrm{eff}}$ : the exact solution at higher $n_{\mathrm{g}}$ approaches the value given by Equation $(19)$, whereas $\Delta T_{\mathrm{g}}$ predicted by the standard model keeps decreasing as $\propto n_{\mathrm{g}}^{-1}$.

The dotted lines in Figure 2 highlight the crucial role of the size distribution, showing $\Delta T_{\mathrm{g}}\left(\zeta_{\text {ion }}\right)$ derived from the standard model where grains are assumed to be monodisperse. Following the original work of Goldsmith 
(2001), we set the grain radius to the fiducial value of $a=0.17 \mu \mathrm{m}$ (while the other parameters entering Equation (18) are kept the same as above). Since this value is a factor of a few larger than $a_{\text {eff }}=0.035 \mu \mathrm{m}$ for the MRN dust, for $n_{\mathrm{g}} \lesssim \times 10^{6} \mathrm{~cm}^{-3}$ the resulting $\Delta T_{\mathrm{g}}$ is systematically overestimated and the dotted line lies well above the solid line (while near $n_{\mathrm{g}} \sim 3 \times 10^{6} \mathrm{~cm}^{-3}$ the discrepancy between the exact and the standard models are almost compensated). In the following section we elaborate on the major effect of the size distribution.

\subsection{Impact of dust evolution}

Possible dust evolution due to ongoing coagulation of grains (see, e.g., Flower et al. 2005, Chacón-Tanarro et al. 2017) should reduce the total surface area of dust and, hence, the thermal coupling with gas, thus increasing $\Delta T_{\mathrm{g}}$. A reduction of the area is described by the effective grain radius, Equation (16), which is proportional to the dust mass-to-area ratio. As we demonstrated in the beginning of Section 4.1 $a_{\text {eff }}$ is a rapidly increasing function of $a_{\min }, a_{\max }$, and $\gamma$.

To describe the impact of dust evolution on the gas temperature and investigate how accurately the effective radius quantifies this, in Figure 3 we plot $\Delta T_{\mathrm{g}}$ as a function of the ratio $A_{0} / a_{\text {eff }}$. We consider a range of gas densities of $10^{5} \mathrm{~cm}^{-3} \leq n_{\mathrm{g}} \leq 3 \times 10^{7} \mathrm{~cm}^{-3}$, representative of very dense pre-stellar cores (Keto \& Caselli 2010 Caselli et al. 2019). This gives the range of the critical radii $A_{0}\left(n_{\mathrm{g}}\right)$, as determined by Equation 13 for $T_{\mathrm{g}}=T_{\mathrm{d} 0}$. Assuming the evolution primarily leads to depletion of smaller grains, we vary $a_{\min }$ in the whole size range of the canonical MRN dust, $0.005 \mu \mathrm{m} \leq a_{\min } \leq 0.25 \mu \mathrm{m}$, while $a_{\max }$ is fixed to the maximum value and $\gamma$ varies between 0 and 1 (Weingartner \& Draine 2001). This yields $a_{\text {eff }}$ varying between $0.035 \mu \mathrm{m}$ (non-evolved MRN dust) and $0.25 \mu \mathrm{m}$ (highly-evolved dust, concentrated at the upper bound of the MRN distribution). The exact values of $\Delta T_{\mathrm{g}}$ (color-coded dots) are then computed from Equations (10) and (11). The approach of effective grain radius, Equations (14) and (17), is represented by the dashed line.

Figure 3 shows that for sufficiently low gas densities, $n_{\mathrm{g}} \lesssim 3 \times 10^{5} \mathrm{~cm}^{-3}$, the resulting values of $A_{0} / a_{\text {eff }}$ are small. Then the exact $\Delta T_{\mathrm{g}}$ does not practically depend on the degree of the dust evolution and tends to the prediction of the generalized standard model - the limit described by Equation (18). The parameters of the size distribution have a fairly weak impact on $\Delta T_{\mathrm{g}}$ also at high densities, $n_{\mathrm{g}} \gtrsim 10^{7} \mathrm{~cm}^{-3}$, where $A_{0} / a_{\text {eff }}$ is large, and the plot tends to the universal asymptote of Equation (19). We note that for $\Delta T_{\mathrm{g}} \lesssim 3 \mathrm{~K}$, where the nonlinearity is negligible, the plot simply scales with $\zeta_{\text {ion }}$.

Figure 3 also highlights the fact that the maximum discrepancy between the approach of effective grain radius and the exact results occurs near $A_{0} / a_{\text {eff }} \sim 1$. The discrepancy does not exceed $30 \%$ for the MRN dust and is naturally reduced for evolved dust, since Equation (17) becomes exact for monodisperse grains. As expected, in the limits of small or large $A_{0} / a_{\text {eff }}$ the exact results converge to the dashed line. ${ }^{4}$

\footnotetext{
4 A marginal deviation of the dashed line from the exact results seen in Figure 3 for $\Delta T_{\mathrm{g}} \gtrsim 3 \mathrm{~K}$ is due to a series expansion of
}

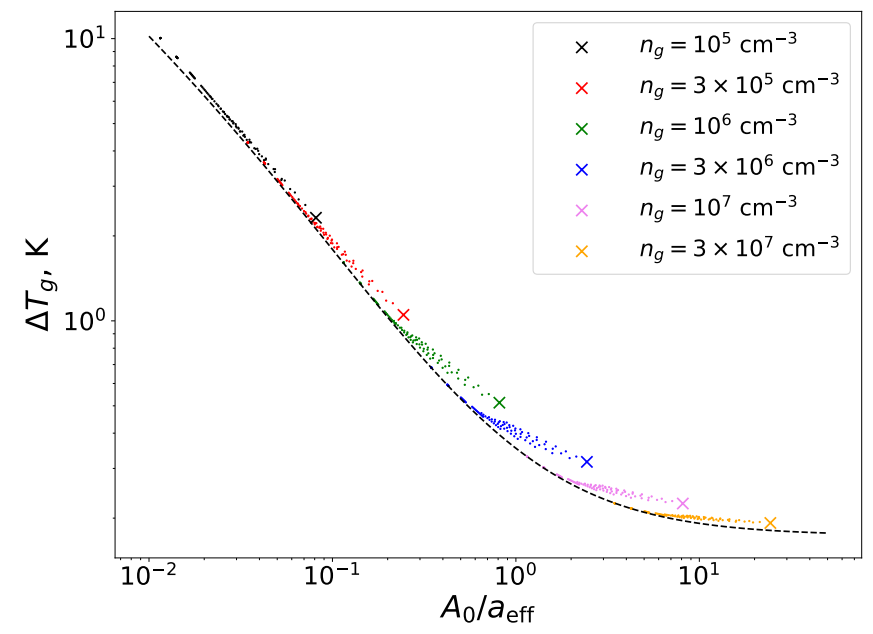

FIG. 3.- Gas temperature difference $\Delta T_{\mathrm{g}}$ versus the ratio $A_{0}\left(n_{\mathrm{g}}\right) / a_{\text {eff }}$. The exact results, Equations 10 and 11 , are plotted for $\zeta_{\text {ion }}=10^{-16} \mathrm{~s}^{-1}$ and for six characteristic values of $n_{\mathrm{g}}$ (color-coded, see the inset). The crosses indicate the non-evolved MRN dust; the dust evolution is quantified by the value of $a_{\mathrm{eff}}$, calculated for varying $\gamma$ and $a_{\min }$ while $a_{\max }$ is fixed (see text for details). The dashed line represents the approach of effective grain radius, Equations 14 and 17.

We recall that evolved dust is comprised of aggregates of smaller solid grains, and thus the effective material density of such aggregates is expected to decrease with size. The effective density can be described by adopting a common approach of fractal dust (see, e.g., Okuzumi et al. 2009, and references therein), where the mass of a particle of radius $a$ is assumed to scale as $\propto a^{D}$, with $2 \lesssim D \lesssim 3$ being the fractal dimension. While the discussion of existing fractal models and the choice of appropriate fractal dimension is beyond the scope of the present paper, we note that our results can, in principle, be generalized for fractal dust, by substituting the corresponding mass scaling into the rhs of Equation (9).

\subsection{Dust emission}

The continuum dust emission is another observable to characterize the processes occurring in dense cores (Chacón-Tanarro et al. 2019). The emission is given by Equation (2) averaged over the grain size distribution. The effective temperature of the emission can thus be defined as

$$
T_{\mathrm{d}, \mathrm{eff}}^{6}=\frac{0.5+\gamma}{\tilde{R}^{0.5+\gamma}-1} \int_{1}^{\tilde{R}} T_{\mathrm{d}}^{6}(x) x^{-0.5+\gamma} d x
$$

where $x=a / a_{\min }$. We obtain $T_{\mathrm{d} \text {,eff }}$ from the energy balance for dust grains, by integrating Equation (1) over the size distribution, equating the resulting rhs to $\Gamma_{\mathrm{CR}}$, and utilizing relation (9):

$$
T_{\mathrm{d}, \mathrm{eff}}^{6}=T_{\mathrm{d} 0}^{6}+\frac{\zeta_{\text {ion }} \varepsilon_{\text {heat }} \rho_{\mathrm{d}}}{3 f_{\mathrm{d}}^{*} m_{\mathrm{g}} q_{\mathrm{abs}} \sigma} .
$$

One can see that Equation 21 is an inversion of Equation 190 with $T_{\mathrm{d} \text {,eff }}$ substituted for $T_{\mathrm{g}}$, and therefore the two temperatures coincide in the limit of large $A_{0} / a_{\text {eff }}$.

$v_{\mathrm{g}}^{*}\left(T_{\mathrm{g}}\right)$, used to derive Equation (17). In this case, the dependence is perfectly described by Equation (18). 
We stress, however, that Equation $\sqrt{19}$ is only applicable in the limit of strong gas-dust coupling, while Equation (21) is appropriate for all conditions where the governing equations of our model are valid. A notable property of $T_{\mathrm{d} \text {,eff }}$ is that it depends neither on gas density nor on the grain size distribution.

To explore how accurately the effective dust temperature represents the emission for different values of $\zeta_{\text {ion }}$, we have computed the total emissivity of grains for varying $n_{\mathrm{g}}$ and parameters of the size distribution (similar to that in Figure 33 and compared the results with the modified black-body radiation of grains at $T_{\mathrm{d}}=T_{\mathrm{d} \text {,eff }}\left(\zeta_{\text {ion }}\right)$. We have found that the deviation of the effective spectral energy distribution from the exact dependence in the Rayleigh-Jeans regime is practically negligible for $\zeta_{\text {ion }} \lesssim 10^{-15} \mathrm{~s}^{-1}$; a weak dependence on $n_{\mathrm{g}}$ is only seen far in the Planck regime (where the emissivity is already decreased by orders of magnitude). As the higher values of $\zeta_{\text {ion }}$ are unlikely in the local cores (Neufeld \& Wolfire 2017), we conclude that the effective temperature determined by Equation (21) provides an excellent universal parametrization of the dust emission.

\subsection{Example: The pre-stellar core L1544}

The physical structure of the pre-stellar core L1544 has been studied in detail in many publications (Tafalla et al. 2002, Crapsi et al. 2007, Keto et al. 2015, ChacónTanarro et al. 2019; Caselli et al. 2019). We have therefore chosen L1544 to illustrate the results of the present theory, and to compare these with the predictions of the commonly used standard theory.

Depending on the particular model of dust opacity used by different authors for calculating the core structure, the dust temperature $\left(T_{\mathrm{d} 0}\right)$ in the core center may vary between 6 and $7 \mathrm{~K}$, while the peak gas density is predicted to be between a few times $10^{6} \mathrm{~cm}^{-3}$ and about $10^{7} \mathrm{~cm}^{-3}$. We adopt the radial distributions for $n_{\mathrm{g}}$ and $T_{\mathrm{d} 0}$ from Keto et al. (2015), with the peak density of $8.3 \times 10^{6} \mathrm{~cm}^{-3}$ and the central dust temperature of $6.3 \mathrm{~K}$ (within a radius of $125 \mathrm{au}$ ), and calculate the dependencies $T_{\mathrm{d} 0}\left(n_{\mathrm{g}}\right)$ and $N\left(n_{\mathrm{g}}\right)$. Then we obtain $\zeta_{\text {ion }}\left(n_{\mathrm{g}}\right)$ from $\zeta_{\text {ion }}(N)$ derived by Padovani et al. (2018b) (their model $\mathscr{H})$, which yields the ionization rate around $10^{-16} \mathrm{~s}^{-1}$ for $10^{5} \mathrm{~cm}^{-3} \lesssim n_{\mathrm{g}} \lesssim 10^{7} \mathrm{~cm}^{-3}$.

Figure 4 summarizes results for the gas and dust temperatures (upper panel) as well as for the CR ionization rate (lower panel), plotted versus the gas density in L1544. The black lines in the upper panel represent different models for $T_{\mathrm{g}}$, calculated for the non-evolved MRN dust. As in Figure 2, we compare the results of our exact theory, Equations (10) and (11), with the generalized standard model, Equation (18), and the standard model for monodisperse grains. We see that the generalized standard model (dashed line) provides a fair description, showing a nearly constant deviation of about $0.25 \mathrm{~K}$ from the exact results (solid line). The reason can be directly understood from Figure 2, where the temperature difference between the solid and dashed lines is, indeed, about that value for $\zeta_{\text {ion }} \sim 10^{-16} \mathrm{~s}^{-1}$. On the other hand, the standard model by Goldsmith (2001); Galli et al. (2002) (dotted line), assuming all grains to have a certain fiducial size (which is significantly larger than $a_{\text {eff }}$ for the MRN dust, see Section 4.1), predicts a gas temperature

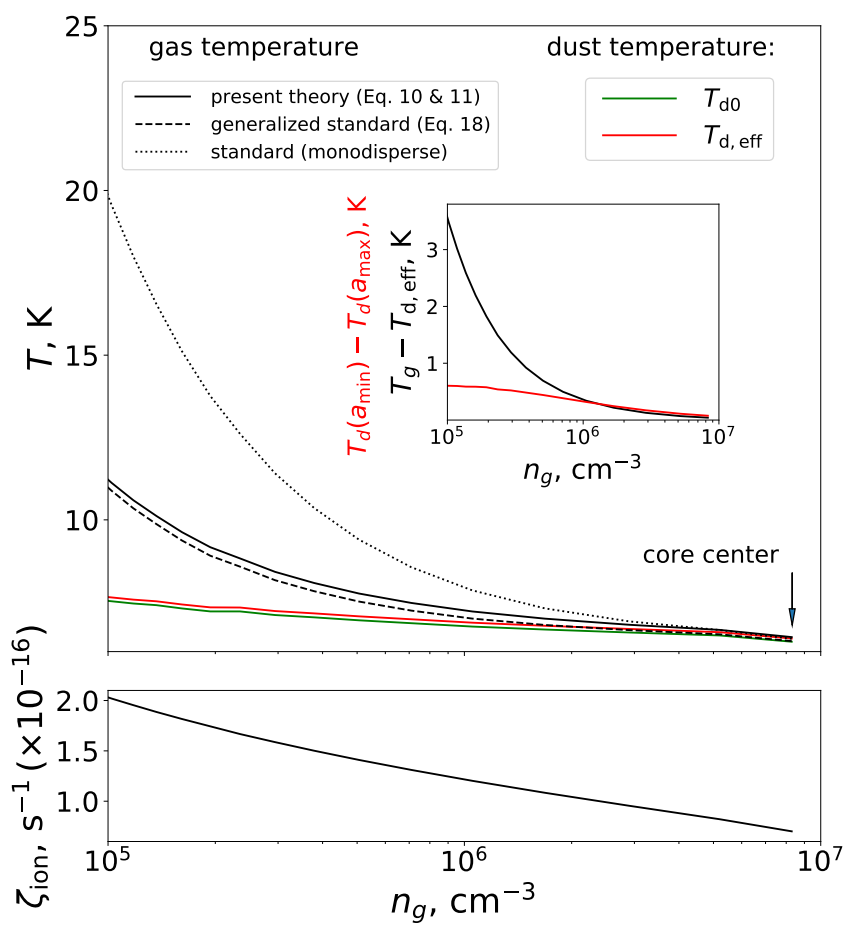

FIG. 4.- Upper panel: Dependence of the gas and dust temperature on the gas volume density $n_{\mathrm{g}}$ in the pre-stellar core L1544. The non-evolved MRN distribution of grain sizes is assumed. For the gas temperature, the legend is the same as in Figure 2 for the dust temperature, the green and red lines represent $T_{\mathrm{d} 0}$ and $T_{\mathrm{d} \text {,eff }}$, respectively. In the inset, we plot the difference between

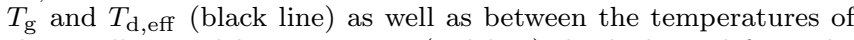
the smallest and largest grains (red line), both derived from the present theory. Lower panel: The $\mathrm{CR}$ ionization rate $\zeta_{\text {ion }}$ versus $n_{\mathrm{g}}$, calculated for L1544 from Padovani et al. (2018b).

that is substantially higher than the results of the present theory up to $n_{\mathrm{g}} \sim 3 \times 10^{6} \mathrm{~cm}^{-3}$. At higher densities, the dotted line goes below the solid line, following the trend seen in Figure2,

Unlike the gas temperature, the effective grain temperature $T_{\text {d.eff }}$ characterizing the continuum emission (see Section 4.3 does not depend on the size distribution. Its deviation from $T_{\mathrm{d} 0}$ remains very small (about $0.1 \mathrm{~K}$ ).

We conclude that for pre-stellar cores in the local ISM, such as L1544, the temperature difference $T_{\mathrm{g}}-T_{\mathrm{d}, \mathrm{eff}}$ is practically equal to the difference $\Delta T_{\mathrm{g}}$, and the latter is reasonably described by the generalized standard theory, Equation (18). The inset in Figure 4 suggests that its magnitude (black solid line) is expected to be between $\approx 3.5 \mathrm{~K}$ and $\approx 0.4 \mathrm{~K}$ for $10^{5} \mathrm{~cm}^{-3} \lesssim n_{\mathrm{g}} \lesssim 10^{6} \mathrm{~cm}^{-3}$, i.e., should be measurable in this density range $\left(T_{\mathrm{g}}-T_{\mathrm{d} \text {,eff }}\right.$ should start decreasing at lower densities, where the line emission becomes the efficient mechanism of gas cooling). Hence, the temperature difference deduced from observational data yields the product $\zeta_{\text {ion }} a_{\text {eff }}$, which should allow us in the future to constrain models of both the $\mathrm{CR}$ ionization and dust evolution in dense cores.

We point out that the present theory, including the generalized standard model, yields gas temperatures in the central region of L1544 which are very close to the measurements (see, e.g., Figures 4(a) and 5 in Crapsi et al. 2007, for $\left.n_{\mathrm{g}} \gtrsim 10^{5} \mathrm{~cm}^{-3}\right)$. A notable fact is that our results are obtained assuming the non-evolved MRN 
distribution of grains and using the ionization rate from Padovani et al. (2018b), who suggest $\zeta_{\text {ion }} \sim 10^{-16} \mathrm{~s}^{-1}$ for this region, whereas the standard theory (monodisperse grains with $\left.a_{\text {eff }} \sim 0.1 \mu \mathrm{m}\right)$ requires $\zeta_{\text {ion }} \sim 10^{-17} \mathrm{~s}^{-1}$ to provide agreement with the measurements (e.g., Crapsi et al. 2007, Keto \& Caselli 2010, Galli \& Padovani 2015). This reflects the crucial role of the grain size distribution (i.e., of a proper choice for $a_{\text {eff }}$ ).

The inset in Figure 4 also demonstrates the difference between the temperatures of the smallest and largest grains, $T_{\mathrm{d}}\left(a_{\min }\right)-T_{\mathrm{d}}\left(a_{\max }\right)$ (red solid line). As follows from Equation $\left(12\right.$, the difference tends to $\approx \Delta T_{\mathrm{g}}$ when the critical radius $A_{0}$ exceeds $a_{\text {min }}$ (the surface $\mathrm{CR}$ heating increases it by the value of $T_{\mathrm{g}, \mathrm{s}}$, see Equations A1 and A3 in Appendix A. For L1544, this occurs at $n_{\mathrm{g}} \gtrsim 4 \times 10^{5} \mathrm{~cm}^{-3}$. At lower densities $T_{\mathrm{d}}\left(a_{\min }\right)-T_{\mathrm{d}}\left(\widetilde{a}_{\max }\right)$ becomes significantly smaller than $\Delta T_{\mathrm{g}}$; its magnitude remains almost constant, reaching $\approx 0.6 \mathrm{~K}$ at $n_{\mathrm{g}}=10^{5} \mathrm{~cm}^{-3}$. This relatively small difference may, nevertheless, have important consequences for the physical and chemical processes occurring on the surface of grains in cold dense cores, due to their extreme temperature dependence.

\section{SUMMARY AND OUTLOOK}

In this paper we showed that the gas temperature $T_{\mathrm{g}}$ in dense pre-stellar cores strongly depends on parameters of the grain size distribution. Furthermore, at high gas densities the value of $T_{\mathrm{g}}$ can substantially exceed the predictions of the standard theory, in which the dust temperature $T_{\mathrm{d}}$ is assumed to be size-independent and equal to $T_{\mathrm{d} 0}(N)$ - the value determined from the balance of radiative heating and cooling of a grain at the column density $N$. Equations (14) and (15) yield an accurate analytical relation between $T_{\mathrm{g}}$ and the $\mathrm{CR}$ ionization rate $\zeta_{\text {ion }}(N)$ for a given size distribution, valid for gas densities $n_{\mathrm{g}} \gtrsim 10^{5} \mathrm{~cm}^{-3}$ (where the gas cooling due to molecular line emission is negligible) and $\lesssim 10^{10} \mathrm{~cm}^{-3}$ (where the coupling to the local thermal radiation of dust can be safely neglected).

We also derived an expression for the effective dust temperature $T_{\text {d,eff }}$, Equation (21), which provides a parametrization of the spectral energy distribution of the continuum emission (valid for the same range of $n_{\mathrm{g}}$ ). We found that the dust emissivity does not depend on the gas density or the grain size distribution, and is solely determined by the values of $\zeta_{\text {ion }}$ and $T_{\mathrm{d} 0}$.

To facilitate the use of our model, in Appendix C we present a convenient parametrization for the gas and dust temperatures, applicable for typical conditions in dense cores:

1. Gas temperature. Equation (C1) gives the relation between $T_{\mathrm{g}}$ and $\zeta_{\text {ion }}$, and also depends on the local parameters $n_{\mathrm{g}}$ and $T_{\mathrm{d} 0}$ as well as on $\tilde{R}$, the relative width of the size distribution. In particular, for $\zeta_{\text {ion }}=10^{-16} \mathrm{~s}^{-1}, T_{\mathrm{d} 0}=6 \mathrm{~K}$, and values of $n_{\mathrm{g}}$ chosen for the plot in Figure 3 , this relation yields the median curves through the corresponding color-coded points (varying $\gamma$ only leads to a slight scatter of the points off the curves, and therefore has a minor effect on the results). The curves connect the crosses (MRN dust) with the dashed line (monodisperse grains) in that figure, thus parameterizing the dependence of $\Delta T_{\mathrm{g}}$ on the size distribution.

2. Effective dust temperature. Equation (C2), relat-

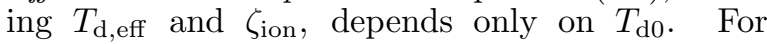
$\zeta_{\text {ion }}=10^{-16} \mathrm{~s}^{-1}$ and $T_{\mathrm{d} 0}=6 \mathrm{~K}$, this yields $T_{\mathrm{d} \text {,eff }}-T_{\mathrm{d} 0} \approx 0.2 \mathrm{~K}$; as expected, this value coincides with the asymptotic value of $\Delta T_{\mathrm{g}}$ in the limit of large $A_{0} / a_{\text {eff }}$ in Figure 3

Our findings imply that measuring both $T_{\mathrm{g}}$ and $T_{\mathrm{d} \text {,eff }}$ in a range of gas densities between $10^{5} \mathrm{~cm}^{-3} \lesssim n_{\mathrm{g}} \lesssim$ $10^{6} \mathrm{~cm}^{-3}$ (where the predicted magnitude of $\Delta T_{\mathrm{g}}$ is expected to be above the measurement uncertainty, see Figure 4), one could develop a method for estimating and constraining the ionization rate and the degree of dust evolution. The present theory predicts the values of $T_{\mathrm{g}}$ in the pre-stellar core L1544 which are very close to the measured values (Crapsi et al. 2007), assuming the nonevolved MRN distribution of grain sizes and the ionization rate as high as $\zeta_{\text {ion }} \sim 10^{-16} \mathrm{~s}^{-1}$. In the future we plan to carry out a detailed analysis and check if our results could reproduce the ALMA and JVLA observations of Caselli et al. (2019) and Crapsi et al. (2007) toward L1544. In general, by combining our model with models for initial stages of dust coagulation in dense pre-stellar cores (Flower et al. 2005; Chacón-Tanarro et al. 2017), we will gain deeper insights into fundamental physical processes occurring in these objects and better understand the mechanisms controlling CR penetration into the clouds. For example, comparing the $\mathrm{CR}$ ionization in diffuse and dense regions of molecular clouds should allow us to discriminate between different transport regimes of CRs, resulting in largely different attenuation of $\zeta_{\text {ion }}$ with the column density (Silsbee \& Ivlev 2019).

Finally, the conclusion that the temperature of smaller grains approaches $T_{\mathrm{g}}$ at higher gas densities, while bigger grains are at $T_{\mathrm{d}} \approx T_{\mathrm{d} 0}$ may have a profound impact on the speed of physical and chemical processes occurring on the dust surface. The thermally activated desorption of atoms and molecules from the surface as well as the surface diffusion and, hence, the diffusion-limited chemical reactions obey the Arrhenius temperature dependence, with the typical activation energy of the order of hundreds of Kelvin (e.g., Vasyunin et al. 2017). Therefore, the temperature increase by only a few tenths of Kelvin for smaller grains (dominating the dust surface) could lead to a significant acceleration of these processes. Furthermore, given a highly uncertain "average" rate of the CR ionization in the ISM (Indriolo \& McCall 2012), and the fact that the ionization can be very strongly enhanced close to protostars (Ceccarelli et al. 2014 Podio et al. 2014) and supernova remnants (Vaupre et al. 2014) - serving as sources of the local CRs, the surface chemistry could be affected dramatically. The additional dust heating by CRs, discussed in Appendix A, could play especially important role in these environments. This problem will be studied in a separate paper.

We would like to thank Daniele Galli for useful discussions and suggestions, and an anonymous referee for constructive and stimulating suggestions. 


\section{APPENDIX \\ APPENDIX A \\ ADDITIONAL DUST HEATING BY CRS}

The CR heating of dust contains both surface (UV absorption, recombination) and volume (IR absorption, CR bombardment) terms. Hence, a sum $\tau a^{2}+v a^{3}$ should be added to the rhs of Equation (1), generalizing the energy balance for a grain. The numerical factors are conveniently determined from

$$
\begin{aligned}
& \tau \int a^{2} d n_{\mathrm{d}}=\zeta_{\text {ion }} \varepsilon_{\mathrm{s}} n_{\mathrm{g}}, \\
& v \int a^{3} d n_{\mathrm{d}}=\zeta_{\text {ion }} \varepsilon_{\mathrm{v}} n_{\mathrm{g}},
\end{aligned}
$$

where $\varepsilon_{\mathrm{s}, \mathrm{v}}$ are the energies per $\mathrm{H}_{2}$ ionization for the surface and volume heating, respectively (see below). We immediately infer that the governing relation between the gas and dust temperatures, Equation (10), remains unchanged after the following replacement:

$$
\begin{aligned}
T_{\mathrm{g}} & \rightarrow T_{\mathrm{g}}+T_{\mathrm{g}, \mathrm{s}}, \\
T_{\mathrm{d} 0}^{6} & \rightarrow T_{\mathrm{d} 0}^{6}+T_{\mathrm{dv}}^{6} .
\end{aligned}
$$

The respective "shifts" due to the surface and volume heating is then obtained from Equation 10,

$$
\begin{aligned}
T_{\mathrm{g}, \mathrm{s}} & =\sqrt{\frac{\pi}{18}} \frac{\zeta_{\text {ion }} \varepsilon_{\mathrm{s}} \rho_{\mathrm{d}} a_{\mathrm{eff}}}{\alpha_{\mathrm{g}} f_{\mathrm{d}}^{*} m_{\mathrm{g}} n_{\mathrm{g}} v_{\mathrm{g}}^{*} k_{\mathrm{B}}}, \\
T_{\mathrm{d}, \mathrm{v}}^{6} & =\frac{\zeta_{\text {ion }} \varepsilon_{\mathrm{v}} \rho_{\mathrm{d}}}{3 f_{\mathrm{d}}^{*} m_{\mathrm{g}} q_{\mathrm{abs}} \sigma},
\end{aligned}
$$

where $a_{\text {eff }}$ is the effective grain radius, Equation (16). Replacing $T_{\mathrm{g}}$ with its shifted value in Equation (11), we conclude that this governing equation remains unchanged, too, if the ionization rate is replaced with

$$
\zeta_{\text {ion }} \rightarrow \zeta_{\text {ion }}\left(1+\frac{\varepsilon_{\mathrm{s}}}{\varepsilon_{\text {heat }}}\right) \text {. }
$$

Thus, Equations (A1)-A5 extend the results of the present paper by including additional CR-induced mechanisms of dust heating.

Note that $T_{\mathrm{g}}$ in governing equations 10 and 11 is to be replaced with its shifted value only where it explicitly enters, i.e., Equation (A1) does not apply to the thermal velocity scale $v_{\mathrm{g}}^{*}\left(T_{\mathrm{g}}\right)$. Consequently, after substituting Equations (A1)- A5 into the analytical approximation, Equations (14) and (15), term $I_{2} \Delta T_{\mathrm{g}}$ in $\Psi\left(\Delta T_{\mathrm{g}}\right)$ splits into two: the first two components of $I_{2}$ in Equation (B1) multiplied with $\Delta T_{\mathrm{g}}$ plus the last component multiplied with $\left(\Delta T_{\mathrm{g}}+T_{\mathrm{g}, \mathrm{s}}\right)$. Similarly, for the approach of effective grain radius, Equation $\left.\sqrt{17}\right)$, term $(\ldots+\ldots) \Delta T_{\mathrm{g}}$ inside the square brackets splits into the first component in the parentheses multiplied with $\Delta T_{\mathrm{g}}$ plus the second component multiplied with $\left(\Delta T_{\mathrm{g}}+T_{\mathrm{g}, \mathrm{s}}\right)$.

The above analysis allows us to understand the relative importance of the surface and volume dust heating by $\mathrm{CRs}$, depending on the value of the critical grain radius $A$. From Equation 12 it follows that the effect of surface heating is weak for grains much larger than $A$, i.e., their temperature is close to $\left(T_{\mathrm{d} 0}^{6}+T_{\mathrm{dv}}^{6}\right)^{1 / 6}$ and the contribution of $T_{\mathrm{g}, \mathrm{s}}$ is negligible. Hence, for $A_{0} \ll a_{\text {eff }}$ the gas temperature is determined from the generalized standard model, Equation (18), with $T_{\mathrm{d} 0}$ replaced according to Equation (A2). In the opposite limit of $A_{0} \gg a_{\text {eff }}$ one can rigorously show that $T_{\mathrm{g}, \mathrm{s}} \lesssim\left(a_{\mathrm{eff}} / A_{0}\right) \Delta T_{\mathrm{g}}$, i.e., $T_{\mathrm{g}, \mathrm{s}}$ is negligible, too. The gas temperature in this case is described by Equation $(19)$ with $T_{\mathrm{d} 0}$ and $\zeta_{\text {ion }}$ replaced according to Equations $(\mathrm{A} 2)$ and $(\mathrm{A} 5)$. This shows that the surface dust heating by CRs is only important for large $A_{0} / a_{\text {eff }}$, where its effect is merely equivalent to increasing the ionization rate.

\section{Role of the additional heating for dense cores}

In Section 4.4 we show that the generalized standard model reasonably describes $T_{\mathrm{g}}$ for conditions of the prestellar core L1544, in the density range where the predicted difference between gas and dust temperatures should be measurable. Therefore, for such cores the gas temperature practically does not depend on the surface heating; $T_{\mathrm{g}}$ could only be affected by the additional volume heating, which leads to higher $T_{\mathrm{d} 0}$ according to Equation (A2).

The absorption efficiency of the Lyman-Werner photons by silicate grains with $a \gtrsim 0.01 \mu \mathrm{m}$ is approximately described by a size-independent $Q_{\text {abs }}$ (Draine 2011). Thus, UV radiation due to $\mathrm{H}_{2}$ and He electronic excitation by CRs mostly contributes to the surface heating, with $\varepsilon_{\mathrm{s}} \approx 8 \mathrm{eV}$ per $\mathrm{H}_{2}$ ionization (Dalgarno et al. 1999; Glassgold et al. 2012). (Formation of molecular hydrogen on grains adds to the surface heating, but the resulting value of $\varepsilon_{\mathrm{s}}$ (of the order of a few eV) is quite uncertain.) Near-IR radiation from vibrationally excited $\mathrm{H}_{2}$ could potentially contribute to $\varepsilon_{\mathrm{v}}$ (neglecting collisional quenching, see Dalgarno et al. 1999), with up to $\approx 3 \mathrm{eV}$ per $\mathrm{H}_{2}$ ionization, while the effect of direct CR bombardment is negligible (unless $\zeta_{\text {ion }}$ is extremely high, see Hocuk et al. 2017). By comparing Equation (A4) with 21, noting that $\varepsilon_{\mathrm{v}} / \varepsilon_{\text {heat }}<0.2$, and making use of parametrization (C2) in Appendix C we conclude that the expected correction to $T_{\mathrm{d} 0}^{6}$ cannot exceed a few percent. 


\section{APPENDIX B}

FUNCTIONS $I_{1,2}$

Auxiliary functions $I_{1,2}(\tilde{A}, \tilde{R}, \gamma)$ entering Equation 15$)$ are given by the following expressions:

$$
I_{1}=\int_{1}^{\tilde{R}} \frac{x^{-0.5+\gamma}}{x+\tilde{A}} d x, \quad I_{2}=\frac{1}{2}\left(I_{1}+\tilde{A} \frac{\partial I_{1}}{\partial \tilde{A}}+\frac{5}{2} \tilde{A}^{2} \frac{\partial^{2} I_{1}}{\partial \tilde{A}^{2}}\right) .
$$

For the MRN distribution $(\gamma=0)$ we get

$$
I_{1}=2 \frac{\arctan \sqrt{\tilde{A}}-\arctan \sqrt{\tilde{A} / \tilde{R}}}{\sqrt{\tilde{A}}} .
$$

Generally, the integral in Equation (B1) can be calculated analytically for integer and half-integer $\gamma$.

\section{APPENDIX C
PARAMETRIZATION FOR $T_{\mathrm{G}}$ AND $T_{\mathrm{D}, \mathrm{EFF}}$}

To calculate the gas temperature, we employ the general analytical approximation, Equations (14) and (15), with $I_{1}$ from Equation (B2). Figure 3 suggests that for typical conditions in dense cores the nonlinearity in $T_{\mathrm{g}}$ is only significant for small values of $A_{0} / a_{\mathrm{eff}}$, asymptotically described by Equation $(18)$. Therefore, we use $v_{\mathrm{g}}^{*}\left(T_{\mathrm{g}}\right)$ for the thermal velocity scale in Equation (14) and neglect the term with $I_{2}$ in Equation (15); the latter introduces only a small error (of about $6 \%$ for $\zeta_{\text {ion }}=10^{-16} \mathrm{~s}^{-1}$ ) in the regime of large $A_{0} / a_{\text {eff }}$. After some manipulation, we obtain

$$
\left(T_{\mathrm{g}}-T_{\mathrm{d} 0}\right) \sqrt{T_{\mathrm{g}}}=\left(\frac{\zeta_{\text {ion }}}{10^{-16} \mathrm{~s}^{-1}}\right)\left(\frac{q}{\arctan q}\right) \frac{1+p_{2}}{p_{1}},
$$

where $T_{\mathrm{g}}$ and $T_{\mathrm{d} 0}$ are in units of Kelvin. Equation (C1) depends on the following three parameters:

$$
\begin{aligned}
p_{1} & =0.0252 \sqrt{\tilde{R}}\left(\frac{n_{\mathrm{g}}}{10^{5} \mathrm{~cm}^{-3}}\right), \\
p_{2} & =0.0115 \sqrt{\tilde{R}}\left(\frac{n_{\mathrm{g}}}{10^{5} \mathrm{~cm}^{-3}}\right)\left(\frac{T_{\mathrm{d} 0}}{6}\right)^{-5}, \\
q & =\frac{\sqrt{p_{2}}}{1+p_{2}} \frac{\sqrt{\tilde{R}}-1}{\sqrt[4]{\tilde{R}}},
\end{aligned}
$$

determined by $\tilde{R}=a_{\max } / a_{\min }$, where $a_{\max }=0.25 \mu \mathrm{m}$ is fixed. Parameter $q$ is a measure of the size distribution width; the case of monodisperse dust is recovered in the limit $q \rightarrow 0$, where $(q / \arctan q) \rightarrow 1$. For $p_{2} \ll 1$, Equation (C1) tends to the generalized standard model, Equation (18), for $p_{2} \gtrsim 1$ it approaches the universal asymptote of Equation (19).

The effective dust temperature is directly obtained from Equation (21):

$$
T_{\mathrm{d}, \mathrm{eff}}=T_{\mathrm{d} 0}\left[1+0.202\left(\frac{\zeta_{\text {ion }}}{10^{-16} \mathrm{~s}^{-1}}\right)\left(\frac{T_{\mathrm{d} 0}}{6}\right)^{-6}\right]^{1 / 6},
$$

where, again, $T_{\mathrm{d}, \mathrm{eff}}$ and $T_{\mathrm{d} 0}$ are in units of Kelvin.

\section{REFERENCES}

Akimkin, V., Zhukovska, S., Wiebe, D., et al. 2013, ApJ, 766, 8

Bacalla, X. L., Linnartz, H., Cox, N. L. J., et al. 2019, A\&A, 622, A31

Bergin, E. A., \& Tafalla, M. 2007, ARA\&A, 45, 339

Bonnor, W. B. 1956, MNRAS, 116, 351

Burke, J. R., \& Hollenbach, D. J. 1983, ApJ, 265, 223

Caselli, P., Walmsley, C. M., Tafalla, M., Dore, L., \& Myers, P. C. 1999, ApJ, 523, L165

Caselli, P., Pineda, J. E., Zhao, B., et al. 2019, ApJ, 874, 89

Ceccarelli, C., Dominik, C., López-Sepulcre, A., et al. 2014, ApJ, $790, \mathrm{~L} 1$

Cecchi-Pestellini, C., \& Aiello, S. 1992, MNRAS, 258, 125

Chacón-Tanarro, A., Caselli, P., Bizzocchi, L., et al. 2017, A\&A, 606, A142

Chacón-Tanarro, A., Pineda, J. E., Caselli, P., et al. 2019, A\&A, 623, A118

Crapsi, A., Caselli, P., Walmsley, C. M., et al. 2005, ApJ, 619, 379
Crapsi, A., Caselli, P., Walmsley, M. C., \& Tafalla, M. 2007, A\&A, 470, 221

Dalgarno, A., Yan, M., \& Liu, W. 1999, ApJS, 125, 237

Draine, B. T. 1978, ApJS, 36, 595

-. 2011, Physics of the Interstellar and Intergalactic Medium (Princeton: Princeton University Press)

Evans, Neal J., I., Rawlings, J. M. C., Shirley, Y. L., \& Mundy, L. G. 2001, ApJ, 557, 193

Flower, D. R., Pineau Des Forêts, G., \& Walmsley, C. M. 2005, A\&A, 436, 933

Galli, D., \& Padovani, M. 2015, arXiv e-prints, arXiv:1502.03380

Galli, D., Walmsley, M., \& Gonçalves, J. 2002, A\&A, 394, 275

Glassgold, A. E., Galli, D., \& Padovani, M. 2012, ApJ, 756, 157

Goldsmith, P. F. 2001, ApJ, 557, 736

Harju, J., Daniel, F., Sipilä, O., et al. 2017, A\&A, 600, A61

Hocuk, S., Szücs, L., Caselli, P., et al. 2017, A\&A, 604, A58

Indriolo, N., \& McCall, B. J. 2012, ApJ, 745, 91 
Ivlev, A. V., Dogiel, V. A., Chernyshov, D. O., et al. 2018, ApJ, 855,23

Keto, E., \& Caselli, P. 2008, ApJ, 683, 238

—. 2010, MNRAS, 402, 1625

Keto, E., Caselli, P., \& Rawlings, J. 2015, MNRAS, 446, 3731

Keto, E., Rawlings, J., \& Caselli, P. 2014, MNRAS, 440, 2616

Launhardt, R., Stutz, A. M., Schmiedeke, A., et al. 2013, A\&A, 551, A98

Léger, A., Jura, M., \& Omont, A. 1985, A\&A, 144, 147

McKee, C. F. 1989, ApJ, 345, 782

Neufeld, D. A., \& Wolfire, M. G. 2017, ApJ, 845, 163

Okuzumi, S., Tanaka, H., \& Sakagami, M.-a. 2009, ApJ, 707, 1247

Padovani, M., Galli, D., Ivlev, A. V., Caselli, P., \& Ferrara, A. 2018a, A\&A, 619, A144

Padovani, M., Ivlev, A. V., Galli, D., \& Caselli, P. 2018b, A\&A, 614, A111

Podio, L., Lefloch, B., Ceccarelli, C., Codella, C., \& Bachiller, R. 2014, A\&A, 565, A64

Prasad, S. S., \& Tarafdar, S. P. 1983, ApJ, 267, 603

Sadavoy, S. I., Stutz, A. M., Schnee, S., et al. 2016, A\&A, 588, A30

Shen, C. J., Greenberg, J. M., Schutte, W. A., \& van Dishoeck, E. F. 2004, A\&A, 415, 203
Shingledecker, C. N., Tennis, J., Le Gal, R., \& Herbst, E. 2018, ApJ, 861, 20

Shu, F. H., Adams, F. C., \& Lizano, S. 1987, ARA\&A, 25, 23

Silsbee, K., \& Ivlev, A. V. 2019, ApJ, 879, 14

Sipilä, O., \& Caselli, P. 2018, A\&A, 615, A15

Sipilä, O., Caselli, P., Redaelli, E., Juvela, M., \& Bizzocchi, L. 2019, MNRAS, 487, 1269

Steinacker, J., Bacmann, A., Henning, T., \& Heigl, S. 2016, A\&A, 593, A6

Tafalla, M., Myers, P. C., Caselli, P., Walmsley, C. M., \& Comito, C. 2002, ApJ, 569, 815

Vasyunin, A. I., Caselli, P., Dulieu, F., \& Jiménez-Serra, I. 2017, ApJ, 842, 33

Vaupré, S., Hily-Blant, P., Ceccarelli, C., et al. 2014, A\&A, 568, A50

Weingartner, J. C., \& Draine, B. T. 2001, ApJ, 548, 296

Weingartner, J. C., Draine, B. T., \& Barr, D. K. 2006, ApJ, 645, 1188

Woitke, P., Kamp, I., \& Thi, W.-F. 2009, A\&A, 501, 383

Zhao, B., Caselli, P., \& Li, Z.-Y. 2018, MNRAS, 478, 2723

Zucconi, A., Walmsley, C. M., \& Galli, D. 2001, A\&A, 376, 650 